\title{
The 2dF Galaxy Redshift Survey: the luminosity function of cluster galaxies
}

Roberto De Propris, ${ }^{1,2 \star}$ Matthew Colless, ${ }^{1}$ Simon P. Driver, ${ }^{1}$ Warrick Couch, ${ }^{2}$ John A. Peacock, ${ }^{3}$ Ivan K. Baldry, ${ }^{4}$ Carlton M. Baugh, ${ }^{5}$ Joss Bland-Hawthorn, ${ }^{6}$ Terry Bridges, ${ }^{6}$ Russell Cannon, ${ }^{6}$ Shaun Cole,${ }^{5}$ Chris Collins, ${ }^{7}$ Nicholas Cross, ${ }^{4}$ Gavin B. Dalton, ${ }^{8}$ George Efstathiou, ${ }^{9}$ Richard S. Ellis, ${ }^{10}$ Carlos S. Frenk, ${ }^{5}$ Karl Glazebrook, ${ }^{4}$ Edward Hawkins, ${ }^{11}$ Carole Jackson, ${ }^{1}$ Ofer Lahav, ${ }^{8}$ Ian Lewis, ${ }^{8}$ Stuart Lumsden, ${ }^{12}$ Steve Maddox, ${ }^{11}$ Darren S. Madgwick, ${ }^{9}$ Peder Norberg, ${ }^{5}$ Will Percival, ${ }^{3}$ Bruce Peterson, ${ }^{1}$ Will Sutherland ${ }^{6}$ and Keith Taylor ${ }^{10}$

\footnotetext{
${ }^{1}$ Research School of Astronomy and Astrophysics, The Australian National University, Weston Creek, ACT 2611, Australia

${ }^{2}$ Department of Astrophysics and Optics, University of New South Wales, Sydney, NSW 2052, Australia

${ }^{3}$ Institute for Astronomy, University of Edinburgh, Royal Observatory, Edinburgh EH9 $3 \mathrm{HJ}$

${ }^{4}$ Department of Physics and Astronomy, Johns Hopkins University, 3400 North Charles Street Baltimore, MD 21218, USA

${ }^{5}$ Department of Physics, University of Durham, Science Laboratories, South Road, Durham DH1 3 LE

${ }^{6}$ Anglo-Australian Observatory, PO Box 296, Epping, NSW 2121, Australia

${ }^{7}$ Astrophysics Research Institute, Liverpool John Moores University, Twelve Quays House, Egerton Wharf, Birkenhead, L14 1LD

${ }^{8}$ Department of Physics, Keble Road, Oxford OX1 3RH

${ }^{9}$ Institute of Astronomy, University of Cambridge, Madingley Road, Cambridge CB3 OHA

${ }^{10}$ Department of Astronomy, California Institute of Technology, Pasadena, CA 91125, USA

${ }^{11}$ School of Physics and Astronomy, University of Nottingham, University Park, Nottingham NG7 2RD

${ }^{12}$ Department of Physics and Astronomy, E C Stoner Building, Leeds LS2 9JT
}

Accepted 2003 February 6. Received 2003 January 29; in original form 2002 September 1

\begin{abstract}
We have determined the composite luminosity function (LF) for galaxies in 60 clusters from the $2 \mathrm{dF}$ Galaxy Redshift Survey. The LF spans the range $-22.5<M_{b_{\mathrm{J}}}<-15$, and is well fitted by a Schechter function with $M_{b_{\mathrm{J}}}^{*}=-20.07 \pm 0.07$ and $\alpha=-1.28 \pm 0.03\left(H_{0}=\right.$ $\left.100 \mathrm{~km} \mathrm{~s}^{-1} \mathrm{Mpc}^{-1}, \Omega_{M}=0.3, \Omega_{\Lambda}=0.7\right)$. It differs significantly from the field LF, having a characteristic magnitude that is approximately $0.3 \mathrm{mag}$ brighter and a faint-end slope that is approximately 0.1 steeper. There is no evidence for variations in the LF across a wide range of cluster properties: the LF is similar for clusters with high and low velocity dispersions, for rich and poor clusters, for clusters with different Bautz-Morgan types, and for clusters with and without substructure. The core regions of clusters differ from the outer parts, however, in having an excess of very bright galaxies. We also construct the LFs for early (quiescent), intermediate and late (star-forming) spectral types. We find that, as in the field, the LFs of earlier-type galaxies have brighter characteristic magnitudes and shallower faint-end slopes. However, the LF of early-type galaxies in clusters is both brighter and steeper than its field counterpart, although the LF of late-type galaxies is very similar. The trend of faint-end slope with spectral type is therefore much less pronounced in clusters than in the field, explaining why variations in the mixture of types do not lead to significant differences in the cluster LFs. The differences between the field and cluster LFs for the various spectral types can be qualitatively explained by the suppression of star formation in the dense cluster environment, together with mergers to produce the brightest early-type galaxies.
\end{abstract}

Key words: galaxies: formation - galaxies: luminosity function, mass function. 


\section{INTRODUCTION}

The galaxy luminosity function (LF), which describes the number of galaxies per unit volume as a function of luminosity, is a fundamental tool for testing theories of galaxy formation and interpreting observations of galaxies at high redshift for evidence of evolution. Furthermore, precise and accurate measurements of the LF in different environments have the potential to provide important clues as to the role of 'environmental' processes (e.g. dynamical interactions in rich clusters) in determining the properties of the present-day galaxy population.

One of the main legacies of the numerous redshift surveys that have been undertaken in the last decade or more is the wealth of LF measurements for galaxies in the low-density 'field' environment. A compilation and comparison of these various measurements was recently published by Cross et al. (2001). This work showed that while the data were adequately represented by a Schechter function, there were serious discrepancies between the various measurements, with the LFs differing by as much as a factor of 2 at the $L^{*}$ point, and with a scatter of a factor of 10 at $0.01 L^{*}$. These differences are due to a combination of surface brightness selection, colour, aperture effects and local density variations among others. These problems have now been overcome in the recent analysis of large redshift surveys such as the 2dF Galaxy Redshift Survey (2dFGRS; Folkes et al. 1999; Madgwick et al. 2002; Norberg et al. 2002) and the Sloan Digital Sky Survey (SDSS; Blanton et al. 2001).

Rich clusters of galaxies provide the other extreme in environment, representing the highest density regions inhabited by galaxies. It is generally easier to derive LFs in clusters, as they provide rich ensembles of galaxies all at the same distance, whose overdensity with respect to the surrounding field is sufficiently high to efficiently identify members either photometrically, through the statistical removal of foreground and background galaxies (e.g. Driver, Couch \& Phillipps 1998a and references therein) or spectroscopically (Small, Sargent \& Hamilton 1997; De Propris et al. 1998; Adami et al. 2000).

These techniques have been used to measure LFs for individual clusters, which in many cases have been combined to form a 'composite' LF to improve statistics (particularly at the brightest luminosities) and average out systematic uncertainties (Dressler 1978; Lugger 1986, 1989; Colless 1989; Gaidos 1997; Lumsden et al. 1997; Valotto et al. 1997; Rauzy, Adami \& Mazure 1998; Garilli, Maccagni \& Andreon 1999; Paolillo et al. 2001; Goto et al. 2002; Yagi et al. 2002). However, these studies have not been unanimous on the exact form of the LF, with some claiming that there are significant differences between the LFs from cluster to cluster and between cluster and field (Dressler 1978; Lopez-Cruz et al. 1997; Lumsden et al. 1997; Valotto et al. 1997; Garilli et al. 1999; Driver et al. 1998a; Goto et al. 2002), while others find no differences and conclude that galaxies in all environments appear to be drawn from a single, 'universal' LF (Lugger 1986, 1989; Colless 1989; Gaidos 1997; Rauzy et al. 1998; Trentham 1998; Paolillo et al. 2001; Yagi et al. 2002). Table 1 gives a summary of the Schechter function fits from some of these previous studies (all of which are based on the technique of background subtraction, unlike the present work which uses spectroscopic identifications of cluster members). We have transformed magnitudes to $H_{0}=100 \mathrm{~km} \mathrm{~s}^{-1} \mathrm{Mpc}^{-1}$ but we have not changed their cosmology.

With the 2dFGRS (Colless et al. 2001) now complete, there is an opportunity to revisit these issues and simultaneously address the detailed form of the LF in rich clusters and in the field in a consistent manner. High-quality measurements of the field LF based on 2dFGRS data have already been published by Madgwick et al. (2002) and Norberg et al. (2002). In this paper we present an analysis of the LFs of 60 rich clusters, taken from the sample of known clusters within the 2dFGRS survey region that were identified and characterized by De Propris et al. (2002).

The enormity of $2 \mathrm{dFGRS}$ in terms of its size, depth and sky coverage has a number of distinct advantages in comparison to previous LF studies. First, cluster membership is determined unambiguously from spectroscopic redshifts for nearly all galaxies, eliminating the introduction of systematic errors into the derived LFs through field subtraction (Driver et al. 1998b). Secondly, the apparent magnitude limit of 2dFGRS $\left(b_{\mathrm{J}}=19.45\right)$ is sufficiently deep that, at the redshifts covered here $(z<0.11)$, our study extends to $\sim 5$ mag fainter than $M^{*}$ - at least as deep as previous studies. Thirdly, the sheer number of clusters (60) that we can study together with the almost one-in-one sampling of their galaxy populations, provides the level of statistical discrimination needed, particularly at the bright end of the LF (Colless 1989), to detect differences that are of physical interest. Finally, our comparison of the cluster and field LFs is done entirely within the 2dFGRS and hence based on the same input catalogue, galaxy photometry and redshift observations. The field and cluster samples therefore share most of the selection effects and observational biases and the resulting LFs can be compared fairly. We also note that the field LF in Madgwick et al. (2002) is derived from galaxies with $z<0.15$ and is therefore similar to the volume-limited sample of cluster galaxies.

The plan of this paper is as follows. The next section describes our cluster sample and the procedure used for constructing composite LFs. We then present our derived LFs in Section 3, both for the entire sample of clusters and for subsets differentiated on the basis

Table 1. Composite LFs for rich clusters in blue passbands.

\begin{tabular}{lcccc}
\hline Reference & $\mathrm{M}^{*}$ & $\alpha$ & $N_{\text {clusters }}$ & Luminosity range \\
\hline Schechter (1976) & $-19.9 \pm 0.5$ & -1.24 & 13 & $-22.5<M_{J}<-18.5$ \\
Dressler (1978) & $-19.7 \pm 0.5$ & -1.25 & 12 & $-23.5<M_{F}<-18.5$ \\
Colless (1989) & $-20.10 \pm 0.07$ & -1.25 & 14 & $-22.5<M_{J}<-17$ \\
Lumsden et al. (1997) & $-20.16 \pm 0.45$ & $-1.22 \pm 0.20$ & 46 & $-21<M_{b}<-18$ \\
Valotto et al. (1997) & $-20.0 \pm 0.1$ & $-1.4 \pm 0.1$ & 55 & $-21<M_{b}<-17$ \\
Rauzy et al. (1998) & $-19.91 \pm 0.21$ & $-1.50 \pm 0.11$ & 28 & $-21<M_{b}<-17$ \\
Garilli et al. (1999) & $-20.30 \pm 0.10$ & $-0.94 \pm 0.07$ & 65 & $-22.5<M_{g}<-15.0$ \\
Paolillo et al. (2001) & $-20.22 \pm 0.15$ & $-1.07 \pm 0.08$ & 39 & $-24.5<M_{g}<-16.5$ \\
Goto et al. (2002) & $-21.24 \pm 0.11$ & $-1.00 \pm 0.06$ & 204 & $-24<M_{g^{\prime}}<-17$ \\
\hline
\end{tabular}


of velocity dispersion, Bautz-Morgan (B-M) type, richness, and the presence of substructure. In Section 4 we compare our data with previous work and the field. Our results are discussed and summarized in Section 5. We adopt the 'concordance' cosmology with $\Omega_{m}=0.3, \Omega_{\Lambda}=0.7$ and $H_{0}=100$. This is about $0.07 \mathrm{mag}$ brighter than the Einstein-de Sitter model at the mean redshift $(z=$ 0.07 ) of our cluster sample.

\section{CLUSTER SELECTION AND LF CONSTRUCTION}

\subsection{Cluster sample}

The clusters studied here were drawn from the sample of known clusters within the 2dFGRS, constructed by cross-matching the Abell catalogue (Abell 1958; Abell, Corwin \& Olowin 1989), the Automatic Plate Measuring (APM) catalogue (Dalton et al. 1997) and the Edinburgh-Durham Cluster Catalogue (EDCC; Lumsden et al. 1992) with the 2 dFGRS catalogue (De Propris et al. 2002). Our selection was restricted to clusters with $z<0.11-$ in order to sample well below the predicted $M^{*}$ - and those with at least 40 confirmed members within the Abell radius $\left(1.5 h^{-1} \mathrm{Mpc}\right)$. As part of our previous investigation of the redshifts and velocity dispersions of these clusters (De Propris et al. 2002), we used a 'gapping' algorithm to identify their bona fide members. It is these galaxies that we use for our LF construction. Table 2 lists the clusters used in this study and their relevant properties. We only present a few lines here, the full table being available electronically. The total number of clusters used is 60. As per De Propris et al. (2002), only unique names are cited in the ID column in order of preference as Abell, APM and EDCC; cross references to other cluster catalogues are available in a table on the Internet at the Astrophysical Data Centre (http://adc.gsfc.nasa.gov/adc.html). We stress that this is not intended as an homogeneous sample, selected according to welldefined criteria (e.g. X-ray luminosity,) but a selection of the richest nearby clusters, although the sample is likely to be complete in this respect. The 4186 galaxies used here make up about 3 per cent of the total $2 \mathrm{dF}$ sample at $z<0.11$.

For clusters not classified by Abell et al. (1989), we determine a B-M type based on the luminosity distribution of the brightest members. Our cluster data base, upon which the De Propris et al. (2002) study was based, has since been updated to reflect the final survey total of 221000 galaxies.

\subsection{Composite LFs}

The quality of individual cluster LFs varies, depending on the number of members and the completeness of the redshift identification as described in greater detail below. Rather than present LFs for each individual cluster, we derive a 'composite' LF and study its variation in subsamples constructed according to physically meaningful criteria (e.g. cluster mass, dynamical evolutionary status). This approach makes it feasible to look for real differences which are hidden by small number statistics in individual cases.

Composite LFs were built following the prescriptions of Colless (1989), by summing galaxies in absolute magnitude bins and scaling by the richness of their parent cluster. Specifically, the following summation was carried out

$N_{\mathrm{c} j}=\frac{N_{\mathrm{c} 0}}{m_{j}} \sum_{i} \frac{N_{i j}}{N_{i 0}}$,

where $N_{\mathrm{c} j}$ is the number of galaxies in the $j$ th absolute magnitude bin of the composite LF, $N_{i j}$ is the number in the $j$ th bin of the $i$ th cluster LF, $N_{i 0}$ is the normalization used for the $i$ th cluster LF (taken as the corrected number of galaxies brighter than $M_{b_{\mathrm{J}}}=-19$; see below for details), $m_{j}$ is the number of clusters contributing to the $j$ th bin, and $N_{\mathrm{c} 0}$ is the sum of all the normalizations:

$N_{\mathrm{c} 0}=\sum_{i} N_{i 0}$.

The formal errors in $N_{\mathrm{c} j}$ are computed according to

$\delta N_{\mathrm{c} j}=\frac{N_{\mathrm{c} 0}}{m_{j}}\left[\sum_{i}\left(\frac{\delta N_{i j}}{N_{i 0}}\right)^{2}\right]^{1 / 2}$,

where $\delta N_{\mathrm{c} j}$ and $\delta N_{i j}$ are the formal errors in the $j$ th LF bin for the composite and $i$ th cluster, respectively.

For each cluster we count galaxies in bins of absolute magnitude

$M_{b_{\mathrm{J}}}=b_{\mathrm{J}}-\mu-A_{b_{\mathrm{J}}}-K_{b_{\mathrm{J}}}^{z}$,

where $b_{\mathrm{J}}$ is the apparent magnitude, $\mu$ is the distance modulus, $A_{b_{\mathrm{J}}}$ is the extinction and $K_{b_{\mathrm{J}}}^{z}$ is the $k$-correction. In principle, the $k$-correction could be determined from the $\eta$ parameter (Madgwick et al. 2002), but this is only possible for a fraction of the cluster members with adequate signal-to-noise ratio in their spectra. For this reason we adopt a single $k$-correction of the form

$K_{b_{\mathrm{J}}}^{z}=2.6 z+4.3 z^{2}$,

following Madgwick et al. (2002), which is appropriate for the earlytype galaxies which predominate amongst our sample of cluster members (Fig. 1). The average offset between this correction and that for star-forming galaxies is $0.09 \mathrm{mag}$.

Because we do not have redshifts for all galaxies in the cluster fields (the overall completeness is 81 per cent), we correct for this as a function of apparent magnitude. In each apparent magnitude bin (corresponding to the appropriate absolute magnitude bin for each cluster) we count the number of cluster members $N_{\mathrm{C}}$, the number of galaxies with a measured redshift $N_{\mathrm{R}}$ and the number of galaxies in the input catalogue $N_{\mathrm{I}}$. The completeness-corrected number of cluster members in each bin is therefore

$N_{i j}=\frac{N_{\mathrm{C}} N_{\mathrm{I}}}{N_{\mathrm{R}}}$,

which assumes that redshift identification is not biased towards or against galaxies that are members of rich clusters. The size of this correction varies from cluster to cluster, and as a function of magnitude, but is typically small ( $\sim 10-20$ per cent).

Here $N_{\mathrm{I}}$ is a Poisson variable, as it is drawn from an ideal (infinite) distribution, and $N_{\mathrm{C}}$ is a binomial random variable, the number of 'successes' (cluster members) in $N_{\mathrm{R}}$ 'trials' (redshift measurements) with probability of success $N_{\mathrm{C}} / N_{\mathrm{R}}$. Therefore, the errors are given by

$\frac{\delta^{2} N_{i j}}{N_{i j}^{2}}=\frac{\sigma^{2}\left(N_{\mathrm{I}}\right)}{N_{\mathrm{I}}^{2}}+\frac{\sigma^{2}\left(N_{\mathrm{C}}\right)}{N_{\mathrm{C}}^{2}}$,

which simplifies, using the standard binomial error expression, to

$\frac{\delta^{2} N_{i j}}{N_{i j}^{2}}=\frac{1}{N_{\mathrm{I}}}+\frac{1}{N_{\mathrm{C}}}-\frac{1}{N_{\mathrm{R}}}$.

We also determine LFs for different galaxy spectral types. These types are the same as those used by Madgwick et al. (2002) in their analysis of the field LF and are based on a classification parameter $(\eta)$ derived from a principal component analysis of the 2dFGRS spectra. We determine LFs for the early (type 1), mid (type 2) and late (types $3+4$ ) spectral classes (there are too few galaxies to compute separate LFs for the latter two classes). Fig. 1 shows the 
Table 2. Clusters studied in this paper.

\begin{tabular}{|c|c|c|c|c|c|c|c|}
\hline Cluster ID & RA (1950) & Dec. (1950) & B-M type & $c z$ & $\sigma$ & $N_{\text {members }}$ & Completeness \\
\hline A0930 & $10: 04: 30.65$ & $-05: 22: 48.4$ & III & 17316 & 907 & 91 & 0.84 \\
\hline A0954 & 10:11:11.10 & $+00: 07: 40.2$ & II & 28622 & 832 & 49 & 0.72 \\
\hline A0957 & 10:11:05.10 & $-00: 40: 38.9$ & I-II & 13623 & 722 & 88 & 0.71 \\
\hline A1139 & $10: 55: 36.68$ & $+01: 52: 20.9$ & III & 11876 & 504 & 106 & 0.82 \\
\hline A1189 & 11:08:30.14 & $+01: 21: 42.6$ & III & 28824 & 814 & 42 & 0.77 \\
\hline A1200 & 11:10:03.25 & $-02: 56: 27.6$ & III & 24970 & 825 & 62 & 0.83 \\
\hline A1236 & $11: 20: 10.82$ & $+00: 44: 10.0$ & II & 30533 & 589 & 41 & 0.83 \\
\hline A 1238 & $11: 20: 20.36$ & $+01: 23: 19.4$ & III & 22160 & 586 & 85 & 0.82 \\
\hline A1248 & $11: 21: 08.28$ & $-03: 56: 31.4$ & I-II & 16139 & 798 & 44 & 0.82 \\
\hline A1364 & 11:40:55.99 & $-01: 27: 52.8$ & III & 31859 & 600 & 51 & 0.85 \\
\hline A 1620 & $12: 47: 29.78$ & $-01: 16: 07.1$ & III & 25513 & 1095 & 95 & 0.89 \\
\hline A1663 & 13:00:18.05 & $-02: 14: 57.7$ & II & 24827 & 884 & 91 & 0.80 \\
\hline A1692 & 13:09:41.25 & $-00: 39: 59.7$ & II-III & 25235 & 686 & 65 & 0.80 \\
\hline A 1750 & $13: 28: 36.52$ & $-01: 28: 15.9$ & II-III & 25647 & 981 & 78 & 0.62 \\
\hline A 2660 & 23:42:39.94 & $-26: 06: 42.5$ & I-II & 15974 & 845 & 52 & 0.67 \\
\hline A2716 & 00:00:27.51 & $-27: 24: 50.3$ & I-II & 19889 & 660 & 60 & 0.84 \\
\hline A2734 & 00:08:49.47 & $-29: 07: 58.1$ & III & 18646 & 1038 & 127 & 0.91 \\
\hline A 2780 & $00: 27: 35.61$ & $-29: 44: 02.1$ & III & 29987 & 782 & 46 & 0.83 \\
\hline A3027 & $02: 28: 42.26$ & $-33: 19: 27.7$ & III & 23166 & 907 & 91 & 0.65 \\
\hline A3094 & 03:09:16.42 & $-27: 07: 08.4$ & III & 20475 & 774 & 107 & 0.84 \\
\hline A3880 & $22: 25: 04.97$ & $-30: 49: 51.5$ & II & 17258 & 840 & 122 & 0.83 \\
\hline A4012 & $23: 29: 11.30$ & $-34: 19: 50.8$ & II-III & 16230 & 498 & 73 & 0.88 \\
\hline A4013 & $23: 27: 42.53$ & $-35: 13: 21.6$ & III & 16410 & 904 & 85 & 0.81 \\
\hline A 4038 & $23: 44: 59.00$ & $-28: 24: 10.0$ & III & 9077 & 933 & 175 & 0.89 \\
\hline A 4053 & $23: 52: 10.66$ & $-27: 57: 34.6$ & III & 20927 & 994 & 58 & 0.93 \\
\hline S0003 & 00:00:37.68 & $-28: 09: 24.7$ & I & 19293 & 833 & 46 & 0.91 \\
\hline S0006 & 00:02:09.11 & $-30: 45: 42.7$ & I & 8768 & 630 & 53 & 0.90 \\
\hline S0084 & $00: 46: 57.51$ & $-29: 47: 33.7$ & I & 32664 & 807 & 44 & 0.69 \\
\hline S0141 & $01: 11: 26.28$ & $-32: 00: 45.6$ & I & 5793 & 411 & 110 & 0.74 \\
\hline S0160 & $01: 27: 54.67$ & $-33: 09: 41.4$ & I & 20638 & 627 & 54 & 0.90 \\
\hline S0166 & 01:32:06.97 & $-31: 51: 43.3$ & II & 20908 & 511 & 50 & 0.99 \\
\hline S0167 & 01:32:06.91 & $-33: 05: 30.3$ & I & 19792 & 769 & 66 & 0.89 \\
\hline S0258 & $02: 23: 33.21$ & $-29: 50: 26.9$ & II & 18026 & 593 & 87 & 0.72 \\
\hline S0301 & $02: 47: 27.22$ & $-31: 23: 46.7$ & I & 6652 & 608 & 92 & 0.82 \\
\hline S0333 & 03:13:04.34 & $-29: 25: 41.3$ & II & 20042 & 998 & 74 & 0.90 \\
\hline S0340 & 03:17:55.68 & $-27: 11: 45.6$ & II-III & 20281 & 939 & 43 & 0.87 \\
\hline S1043 & $22: 33: 43.18$ & $-24: 36: 05.2$ & I & 11091 & 1345 & 116 & 0.79 \\
\hline S1086 & 23:02:06.51 & $-32: 49: 14.8$ & I-II & 25561 & 509 & 53 & 0.87 \\
\hline S1136 & $23: 33: 38.55$ & $-31: 52: 48.8$ & III & 18688 & 617 & 50 & 0.82 \\
\hline S1142 & $23: 38: 17.18$ & $-30: 32: 47.5$ & II-III & 24425 & 669 & 40 & 0.89 \\
\hline S1165 & $23: 55: 24.91$ & $-30: 08: 40.9$ & I-II & 8920 & 359 & 56 & 0.90 \\
\hline S1171 & $23: 58: 45.20$ & $-27: 41: 54.5$ & II & 8763 & 788 & 53 & 0.90 \\
\hline APM039 & $00: 14: 26.04$ & $-31: 38: 15.7$ & III & 31792 & 559 & 58 & 0.94 \\
\hline APM078 & $00: 27: 44.53$ & $-29: 53: 26.8$ & III & 30079 & 796 & 41 & 0.83 \\
\hline APM268 & $02: 27: 48.57$ & $-33: 23: 55.5$ & III & 23223 & 832 & 97 & 0.62 \\
\hline APM917 & $23: 38: 58.49$ & $-29: 30: 49.6$ & I & 15358 & 503 & 77 & 0.89 \\
\hline APM945 & $23: 56: 27.74$ & $-32: 04: 33.3$ & I & 17982 & 536 & 41 & 0.87 \\
\hline APM954 & 23:58:20.95 & $-28: 44: 30.4$ & III & 18475 & 445 & 67 & 0.91 \\
\hline EDCC069 & $21: 55: 50.75$ & $-28: 42: 15.8$ & I-II & 6528 & 528 & 64 & 0.72 \\
\hline EDCC119 & $22: 13: 32.57$ & $-25: 55: 10.7$ & I & 25546 & 1112 & 43 & 0.84 \\
\hline EDCC142 & $22: 22: 50.88$ & $-31: 27: 17.9$ & I-II & 8411 & 274 & 44 & 0.77 \\
\hline EDCC 142 & $22: 22: 45.48$ & $-31: 18: 50.8$ & II-III & 17437 & 296 & 53 & 0.75 \\
\hline EDCC153 & $22: 29: 25.49$ & $-31: 29: 12.8$ & III & 17452 & 708 & 40 & 0.69 \\
\hline EDCC155 & $22: 29: 22.08$ & $-25: 39: 20.6$ & I-II & 10310 & 714 & 52 & 0.82 \\
\hline EDCC365 & $23: 52: 33.51$ & $-33: 01: 07.7$ & II & 17748 & 524 & 79 & 0.83 \\
\hline EDCC442 & $00: 23: 02.22$ & $-33: 19: 24.5$ & III & 14867 & 763 & 127 & 0.88 \\
\hline EDCC457 & $00: 33: 35.03$ & $-26: 22: 00.2$ & III & 18492 & 977 & 66 & 0.86 \\
\hline EDCC652 & $02: 25: 11.88$ & $-29: 51: 00.7$ & II-III & 17944 & 583 & 64 & 0.81 \\
\hline EDCC661 & 02:29:45.79 & $-32: 11: 30.6$ & III & 24362 & 302 & 48 & 0.86 \\
\hline EDCC664 & $02: 30: 56.67$ & $-33: 02: 58.9$ & I-II & 23750 & 399 & 52 & 0.75 \\
\hline
\end{tabular}




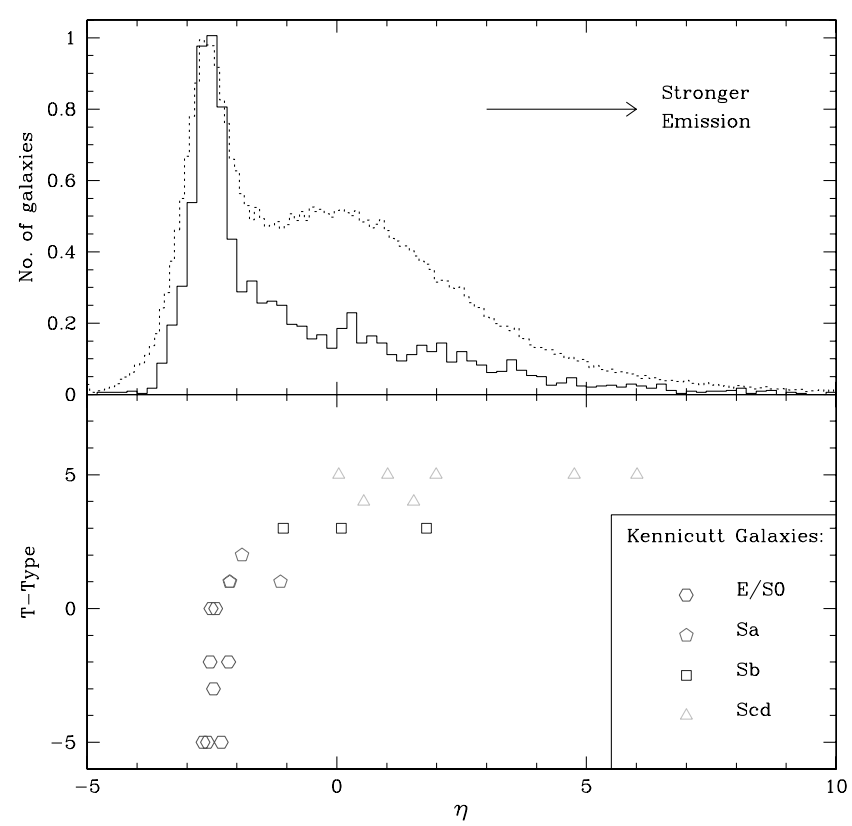

Figure 1. Upper panel: Distribution of $\eta$ parameter values for galaxies in our clusters (solid line) and in the general field (dotted line). Lower panel: The relationship between morphology (represented by T-type) and $\eta$ value as defined by Kennicutt (1992) for nearby galaxies; different symbols are used to indicate the corresponding Hubble types (see legend). It can be seen that early-type galaxies $(\eta \sim-2.5)$ dominate the cluster populations. The relative proportions of type 1, 2, 3, 4 galaxies are $0.54,0.24,0.13,0.09$ in clusters as opposed to $0.36,0.32,0.20,0.12$ in the field, where types are defined as in Madgwick et al. (2002) and in the text.

relationship between spectral type and morphological type and also the distribution of spectral type in both the cluster and field samples. The types are defined as in Madgwick et al. (2002): type 1 galaxies have $\eta<-1.4$, type $2-1.4<\eta<1.1$ and types 3 and $4 \eta>1.1$.

The composite luminosity functions for each spectral type are computed as for the overall sample, except that the completenesscorrected number of galaxies is determined separately for each type as

$N_{i j}(S)=N_{i j} \times \frac{N_{\mathrm{C}}(S)}{\sum_{S} N_{\mathrm{C}}(S)}$,

where $N_{\mathrm{C}}(S)$ is the number of cluster galaxies having spectral type $S(1,2$ or $3+4)$ and the summation is carried out for cluster members over all spectral types. The errors are given by

$\frac{\delta^{2} N_{i j}(S)}{N_{i j}^{2}(S)}=\frac{1}{N_{\mathrm{I}}}+\frac{1}{N_{\mathrm{C}}(S)}-\frac{1}{N_{\mathrm{R}}}$,

where $N_{\mathrm{C}}(S)$ is a binomial variable and $\sum_{S} N_{\mathrm{C}}(S)$ is a Poisson variable, with $k$-corrections for each spectral type as given in Madgwick et al. (2002).

\section{RESULTS}

In Fig. 2, we show the composite LF derived for our complete ensemble of 60 clusters over the full range of absolute magnitude: $-22.5<M_{b_{\mathrm{J}}}<-15$. This LF is based on 4186 cluster members, yielding errors less than 2 per cent over the range $-21.0<M_{b_{\mathrm{J}}}<$ -16.0. A $\chi^{2}$ fit of this LF by a Schechter (1976) function, shown in Fig. 2 as a solid curve, gives a characteristic magnitude of $M_{b_{\mathrm{J}}}^{*}$ $=-20.07 \pm 0.07$ and a faint-end power-law slope of $\alpha=-1.28 \pm$

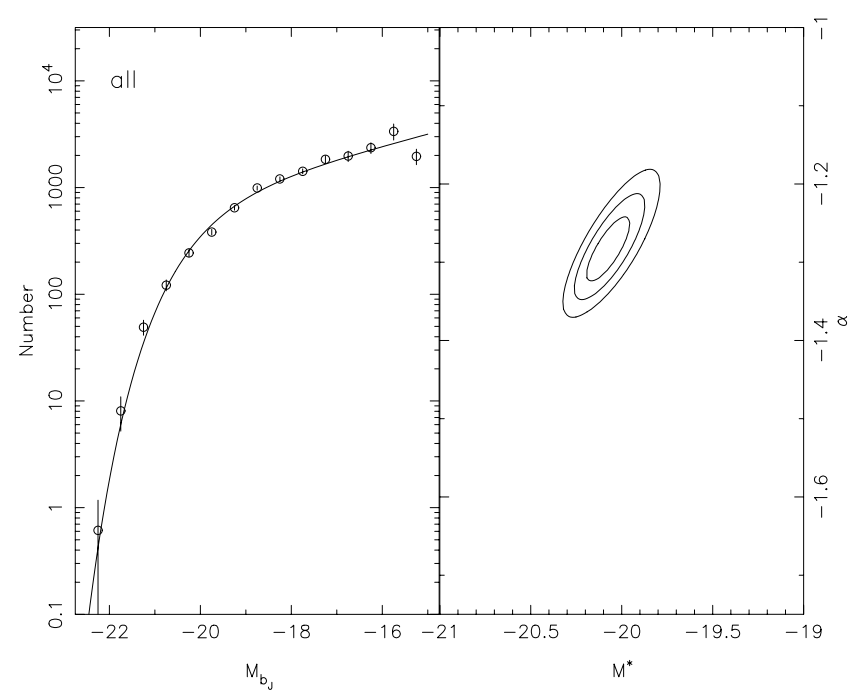

Figure 2. Left-hand panel: The LF of cluster galaxies for the whole sample (open circles), showing the best Schechter function fit (solid line) with parameters as in the text and Table 3. Right-hand panel: 1, 2 and $3 \sigma$ error contours for the best-fitting LF parameters.

0.03 . We tabulate these values in Table 3 together with a measure of the goodness of fit. This fit is only good to about 1 per cent because of the large discrepancies with the last two bins, where completeness corrections are larger and only a few (four) clusters contribute.

Here, as for all our other LFs, the $1 \sigma$ errors are derived by a Monte Carlo simulation, where the independent variable vector is replicated 1000 times, each data point being replaced by the fitted function value plus noise (based on the original error bar for each point), and the function fitted again to the simulated data. The dispersion about the derived $M^{*}$ and $\alpha$ is then used to calculate the errors. The righthand panel of Fig. 2 shows the $\chi^{2}$ error contours corresponding to the 1,2 and $3 \sigma$ levels. It can be seen that the $M^{*}$ and $\alpha$ values are highly correlated, and hence the errors in these quantities quoted in Table 3 are not independent. The fitting procedure is carried out over all three parameters and we marginalized over the normalization to draw these error contours.

We then consider LFs split according to the spectral types described in Madgwick et al. (2002). These types are closely related to the star formation rate and somewhat more loosely to morphology. Our purpose here is to consider the universality of type-specific LFs and how the cluster environment affects star formation. Fig. 3 shows the corresponding data and fits, with error ellipses, for galaxies of spectral types 1,2 and $3+4$.

For purposes of comparison, and to assess the effect of removing the brightest cluster galaxies, we also carry out a fit to the data in Fig. 2 over a restricted absolute magnitude range, $M_{b_{\mathrm{J}}}>-21.5$. This yields a LF with $M_{b_{\mathrm{J}}}^{*}=-20.02 \pm 0.14$ and $\alpha=-1.27 \pm 0.04$ - exclusion of the brightest cluster members thus has no effect on the faint-end slope and leads to a marginally fainter characteristic magnitude, as expected.

We also consider cluster subsamples and plot their LFs and error contours as described below:

(i) We split the sample at a velocity dispersion of $800 \mathrm{~km} \mathrm{~s}^{-1}$, as this is the approximate value at which the distribution of cluster velocity dispersions, $N(\sigma)$, turns over (De Propris et al. 2002), separating massive systems from relatively poor ones. About the same number of galaxies are found in each subsample. 
Table 3. Summary of results.

\begin{tabular}{lcccc}
\hline Sample & $M_{b_{\mathrm{J}}}^{*}$ & $\alpha$ & $P\left(\chi^{2}>\chi_{\text {obs }}^{2}\right)$ & $\mathrm{D} / \mathrm{G}$ ratio \\
\hline All $\left(-22.5<M_{b_{\mathrm{J}}}<-15.5\right)$ & $-20.07 \pm 0.07$ & $-1.28 \pm 0.03$ & 0.013 & $2.09 \pm 0.18$ \\
All $\left(-21.5<M_{b_{\mathrm{J}}}<-16.0\right)$ & $-20.21 \pm 0.11$ & $-1.36 \pm 0.04$ & 0.332 & $2.09 \pm 0.18$ \\
Type 1 & $-20.04 \pm 0.09$ & $-1.05 \pm 0.04$ & 0.000 & $1.29 \pm 0.16$ \\
Type 1 (rich) & $-20.14 \pm 0.12$ & $-1.22 \pm 0.06$ & 0.156 & $1.41 \pm 0.38$ \\
Type 1 (poor) & $-20.06 \pm 0.17$ & $-1.02 \pm 0.07$ & 0.001 & $1.28 \pm 0.14$ \\
Type 2 & $-19.48 \pm 0.13$ & $-1.23 \pm 0.07$ & 0.606 & $2.68 \pm 0.53$ \\
Type 3+4 & $-19.14 \pm 0.19$ & $-1.30 \pm 0.10$ & 0.182 & $4.88 \pm 0.97$ \\
$\sigma>800$ & $-19.99 \pm 0.16$ & $-1.28 \pm 0.05$ & 0.009 & $1.84 \pm 0.23$ \\
$\sigma<800$ & $-20.20 \pm 0.14$ & $-1.35 \pm 0.04$ & 1.000 & $2.11 \pm 0.25$ \\
Rich & $-19.96 \pm 0.12$ & $-1.25 \pm 0.05$ & 0.083 & $1.68 \pm 0.36$ \\
Poor & $-20.29 \pm 0.18$ & $-1.37 \pm 0.05$ & 0.233 & $2.10 \pm 0.15$ \\
B-M I,I-II,II & $-20.11 \pm 0.16$ & $-1.32 \pm 0.05$ & 0.000 & $2.12 \pm 0.21$ \\
B-M II-III,III & $-20.17 \pm 0.15$ & $-1.34 \pm 0.05$ & 0.002 & $1.83 \pm 0.28$ \\
No substructure & $-20.03 \pm 0.12$ & $-1.27 \pm 0.04$ & 0.003 & $1.85 \pm 0.17$ \\
Substructure & $-20.59 \pm 0.31$ & $-1.51 \pm 0.08$ & 0.000 & $3.02 \pm 0.74$ \\
$(r<300$ kpc) & $-20.45 \pm 0.43$ & $-1.44 \pm 0.08$ & 0.000 & $2.02 \pm 0.36$ \\
$(r>300$ kpc) & $-19.83 \pm 0.08$ & $-1.29 \pm 0.05$ & 0.581 & $2.98 \pm 0.25$ \\
& Field (Madgwick et al. 2002) & & & \\
All & $-19.79 \pm 0.04$ & $-1.19 \pm 0.01$ & & \\
Type 1 & $-19.58 \pm 0.05$ & $-0.52 \pm 0.02$ & & \\
Type 2 & $-19.58 \pm 0.03$ & $-0.96 \pm 0.01$ & & \\
Type 3 & $-19.17 \pm 0.04$ & $-1.21 \pm 0.02$ & & \\
Type 4 & $-19.19 \pm 0.04$ & $-1.36 \pm 0.03$ & & \\
Type 3+4 & $-19.14 \pm 0.06$ & $-1.30 \pm 0.03$ & & \\
\hline & & & & \\
& & & &
\end{tabular}

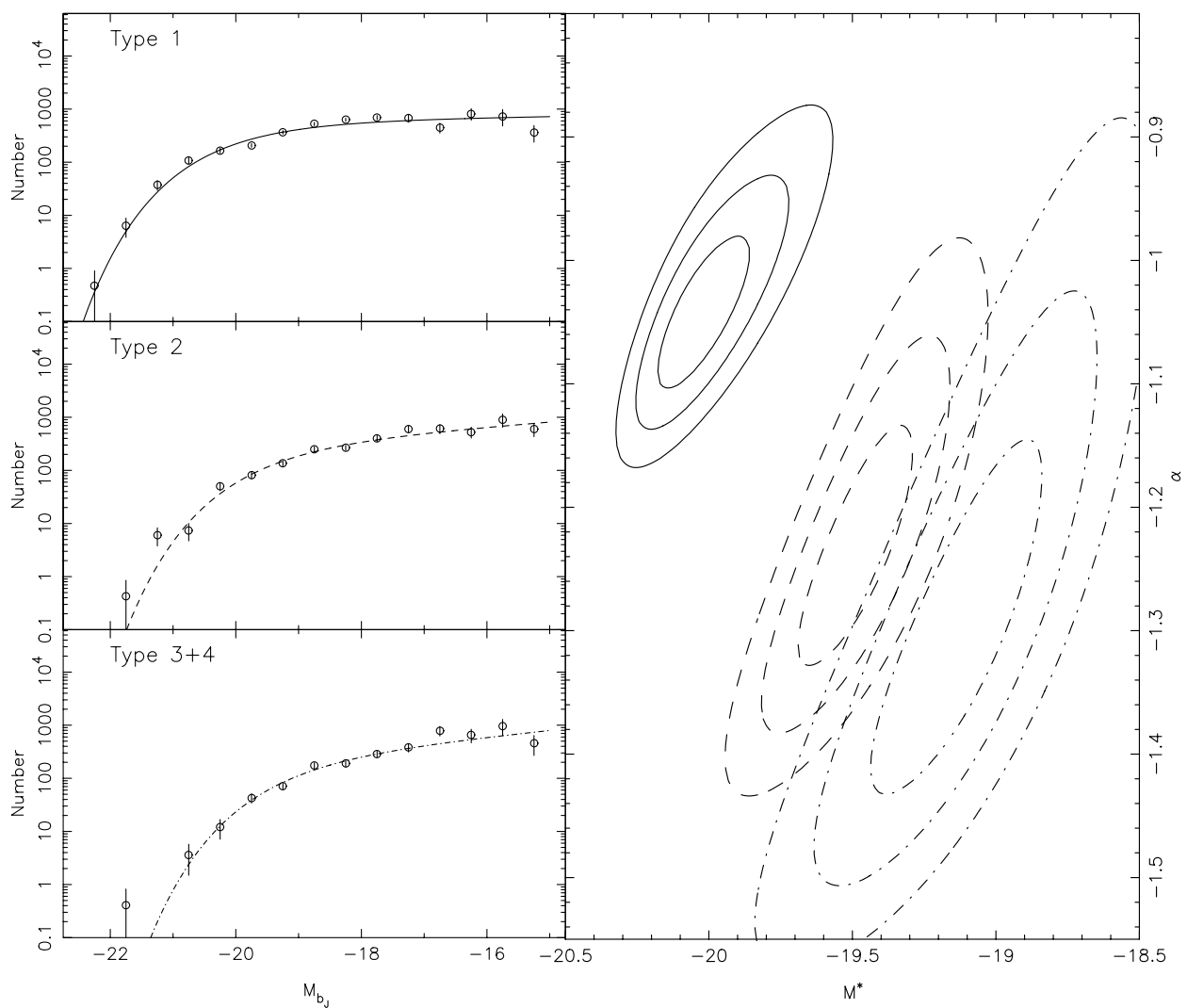

Figure 3. Left-hand panels: LFs for type 1, 2 and 3+4 galaxies (as identified in the panel legends), together with their best-fitting Schechter functions. Right-hand panel: 1,2 and $3 \sigma$ error contours for the Schechter function fits (with the same line styles as in the left-hand panels). 
(ii) We used the normalization parameter, $N_{i 0}$, as a measure of richness, with its median value being used to divide the sample. Here the choice is arbitrary and is mostly motivated by having similar numbers of galaxies in each subset and hence equal statistical weights.

(iii) B-M types measure the dominance of the brightest cluster members over the rest of the cluster population. In the cannibalism model of Ostriker \& Hausman (1978), the brightest cluster galaxies grow at the expense of other less massive galaxies and therefore the LF should vary as the cluster evolves. We chose to divide clusters into two bins: 'early' B-M types, (B-M types I, I-II and II) and 'late' B-M types (having B-M types II-III and III). The former may be evolved systems, whereas the latter may be at an earlier stage of dynamical evolution. Again, this split ensures approximately equal numbers of galaxies in each composite LF.

(iv) The presence of substructure may be an indicator of recent or ongoing cluster merging; substructure can be measured from the three-dimensional (3D) distribution of cluster members, although to some extent the definition of substructure is arbitrary. We use the Lee statistic first applied to clusters by Fitchett (1988), which measures the probability that cluster galaxies form a single group or can be split into two groups in position-velocity space. As most clusters showing substructure consist of two groups, this approach is economical. We arbitrarily defined clusters with substructure to be those where the Lee statistic indicates a greater than 50 per cent probability that they consist of two groups. By this definition, about 25 per cent of our clusters contain substructure, a fraction comparable to the 31 per cent of ENACS clusters showing substructure (Solanes, Salvador-Solé \& González-Casado 1999).

(v) We analyse radial trends by considering galaxies inside and outside two King core radii from the cluster centre. The King core radius is about $150 \mathrm{~h}^{-1} \mathrm{kpc}$ (Adami et al. 1998) and, as the densest region of the cluster, is the most likely to show environmental effects. Our choice of two King radii is again motivated by the need to obtain sufficient statistics (i.e. about half the galaxies in each sample).

LFs for all these subsamples, together with their best-fitting Schechter functions and the associated error ellipses are shown in Fig. 4.

Even with our large sample of clusters, some of the brighter and/or fainter absolute magnitude bins are not well populated in a few of the above subsamples - the brighter bins because very bright galaxies are intrinsically less common, and the fainter bins because of the relatively small numbers of clusters at low redshift. For this reason we carry out our fit over a smaller luminosity range, $-21.5<M_{b_{\mathrm{J}}}$ $<-16.0$, for this part of the analysis. For purposes of comparison, the total LF over the same range has $M_{b_{\mathrm{J}}}^{*}=-20.21 \pm 0.11$ and $\alpha=-1.36 \pm 0.04$.

Table 3 shows the derived values for $M^{*}$ and $\alpha$, and the $1 \sigma$ errors, derived from the Monte Carlo simulations referred to above, for our full sample, the cluster subsamples described in the previous section and the individual spectral types. We also tabulate values for the field LF from Madgwick et al. (2002). Data points for all our LFs are also made available in electronic form in the online version of the journal on Synergy.

\section{DISCUSSION}

We used the simulations of Colless (1989) to analyse the ability of our data to detect differences in LF parameters. We find that our LFs are of sufficient quality to detect differences of $0.15 \mathrm{mag}$ in $M^{*}$ and 0.07 in $\alpha$ at the $1 \sigma$ level, which is an improvement by a factor of more than 2 over previous studies. Furthermore, unlike all previous work, we are not limited by uncertainties in background subtraction. In the following, we compare our results to previous determinations of both the cluster and field LFs and study the universality of the LF with cluster subsamples. Type-specific LFs are also discussed. We finally consider the implications of our findings for models of galaxy formation.

\subsection{Comparison with previous work}

A comparison with previous work is shown in Table 1. Among previous studies only Garilli et al. (1999), Paolillo et al. (2001) and Goto et al. (2002) reach luminosity limits comparable to ours. Garilli et al. (1999) derive $M_{g}^{*}=-20.30 \pm 0.10$ and $\alpha=-0.94 \pm 0.07$. Paolillo et al. (2001) derive $M_{g}^{*}=-20.22 \pm 0.15$ and $\alpha=-1.07 \pm$ 0.08 from their 39 clusters. Once we correct for the colour difference between $g$ and $b_{\mathrm{J}}$ and for the different cosmologies (using the mean redshifts of the cluster sample) the characteristic luminosities of Garilli et al. (1999) and Paolillo et al. (2001) agree with our value within their $2 \sigma$ errors, whereas the two $\alpha$ differ at about $2 \sigma$. The two LFs agree with each other at about the $1.5 \sigma$ level; the difference is only marginally significant.

The LF of Goto et al. (2002) from SDSS data is in considerable disagreement with ours: $M^{*}$ is brighter by about 1.2 mag and $\alpha$ is flatter. This LF disagrees with Colless (1989), Lumsden et al. (1997) and Valotto et al. (1997), all of which use APM data. It also disagrees with the $M^{*}$ and $\alpha$ values of Garilli et al. (1999), and Paolillo et al. (2001) in the $g$ band. One possibility is a systematic offset between APM and SDSS photometry, but this appears to be ruled out by a comparison of SDSS Early Release Data, the Millennium Galaxy Catalogue and $2 \mathrm{dF}$ photometry (Cross et al., in preparation).

Earlier work is generally more limited in luminosity coverage. Values for the Schechter function fits for Colless (1989), Lumsden et al. (1992), Valotto et al. (1997) and Rauzy et al. (1998) are, once we apply the necessary cosmological and filter corrections, in reasonable agreement with our data. With the exception of Goto et al. (2002), the agreement with previous work is generally satisfactory.

\subsection{Cluster versus field}

One of the motivations behind this work and previous studies is to test the universality of the LF and its dependence on the environment. The most dramatic comparison in this context is between rich clusters and the field, because of the factor of 100 or more difference in galaxy density between the two environments.

Having derived a statistically robust composite cluster LF from the 2dFGRS data, of foremost interest now is to make this comparison with its 2dFGRS counterpart for the field. We base this comparison on the LFs published by Madgwick et al. (2002); these cover the absolute magnitude range $-23.0<M_{b_{\mathrm{J}}}<-13.5$, and include an overall field LF, and LFs derived by dividing the galaxies into four different spectral classes (see Fig. 1). The $M_{b_{\mathrm{J}}}^{*}$ and $\alpha$ parameter values for their Schechter function fits to these LFs are listed at the bottom of Table 3 . We also compare these LFs directly using a twosample $\chi^{2}$ test, the results of which, for both $M_{b_{\mathrm{J}}}<-16.0$ (the full magnitude range) and $M_{b_{\mathrm{J}}}<-18$ (the brighter cluster members), are shown in Table 4.

The error ellipses for the Schechter function fits to all these LFs are compared in Fig. 5. We only show the $3 \sigma$ contour for field galaxies as the errors are small.

The $\chi^{2}$ comparison shows that the overall cluster and field LFs differ at more than $3 \sigma$, and this conclusion is also borne out by a 

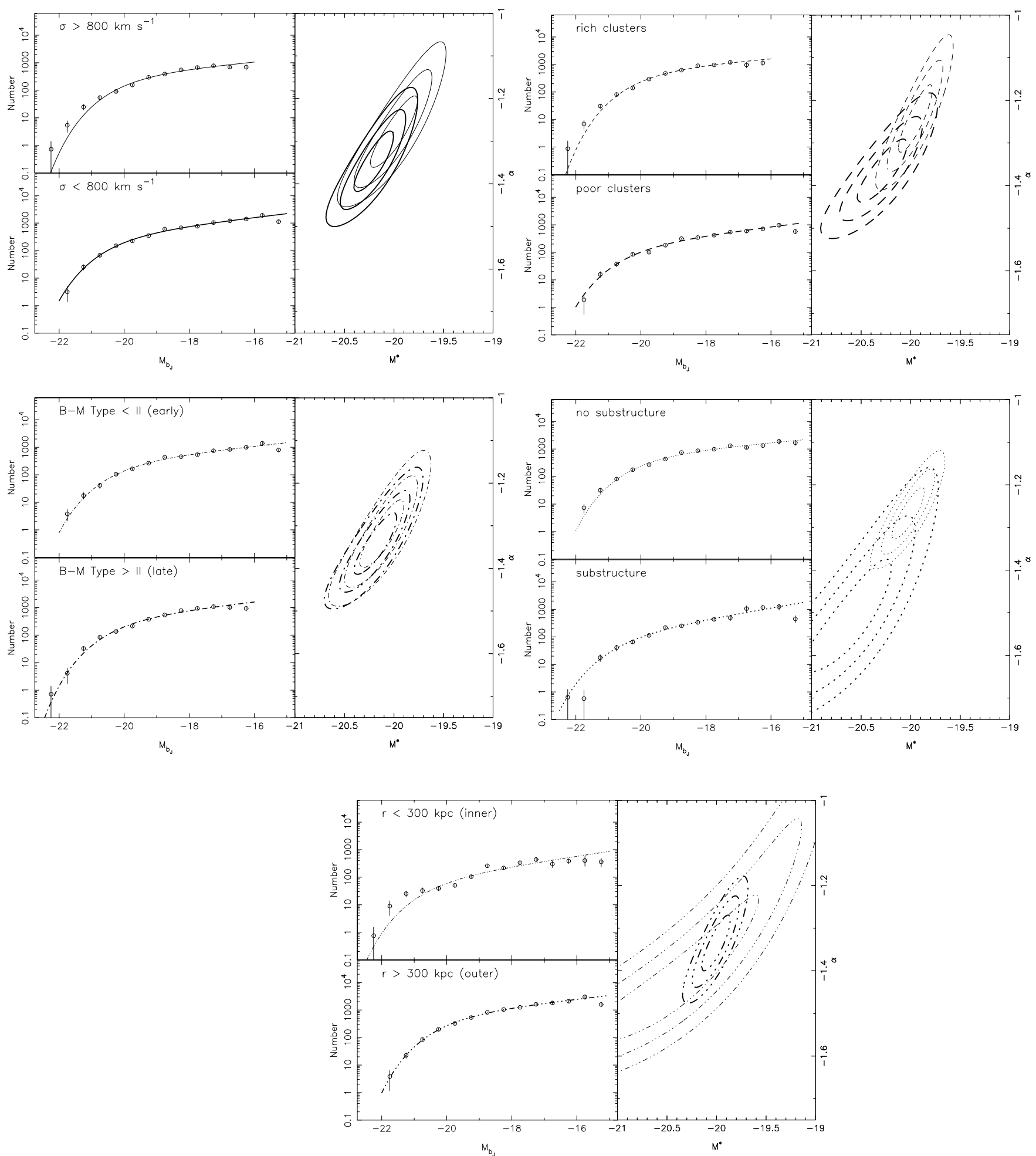

Figure 4. LFs for each cluster subsample, with best-fitting Schechter functions and error contours. The LFs are identified in the legends for each panel and the error ellipses have the same line style as their parent LFs. For example, the thin solid line in the upper left-hand panel shows the LF for galaxies in clusters with high velocity dispersion, while the thick solid line is for clusters with low velocity dispersion.

comparison of the error contours presented in Fig. 5. In terms of the individual LF parameters, the overall cluster LF is 0.3 mag brighter in $M^{*}$ and 0.1 steeper in $\alpha$ than the overall field LF. We note here that, although the 'field' LF is actually the total LF for all environments, clusters do not contribute to the field sample to any great extent, as cluster members are only 3 per cent of the total number of galaxies in the survey; even for bright galaxies this fraction is less than 6 per cent.

Significant differences are also found when the field and cluster LFs for the different spectral classes are compared. For ease of 
Table 4. $\chi^{2}$ comparisons of the field and cluster LFs.

\begin{tabular}{lcc}
\hline Sample & $P\left(\chi^{2}\right)$ & $P\left(\chi^{2}\right)$ \\
& $M_{b_{\mathrm{J}}}<-18$ & $M_{b_{\mathrm{J}}}<-16$ \\
\hline All & $<10^{-3}$ & $<10^{-3}$ \\
Type 1 & $<10^{-3}$ & $<10^{-3}$ \\
Type 2 & 0.133 & $<10^{-3}$ \\
Type 3+4 & 0.556 & 0.320 \\
\hline
\end{tabular}

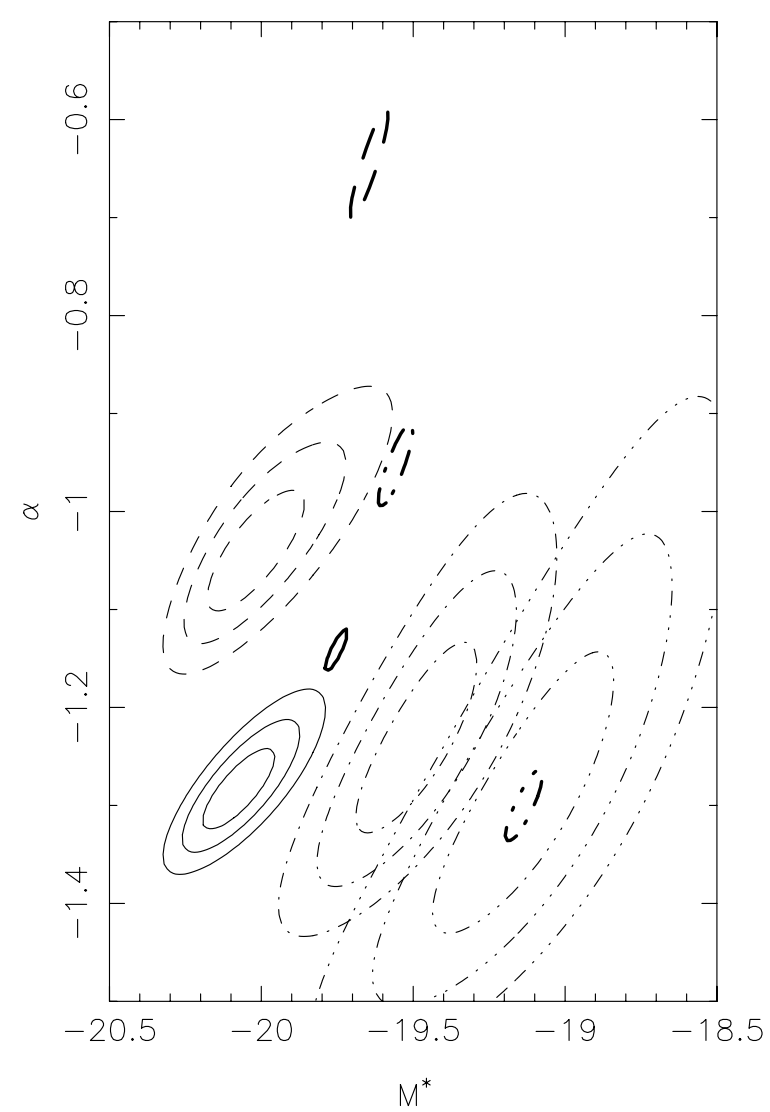

Figure 5. Error ellipses for the Schechter function fits to the field and cluster LFs. The total LFs are represented by solid lines (thin for clusters and thick for the field, as in Fig. 4). Dashed lines are for type 1 galaxies, dot-dashed lines for type 2 galaxies and dotted-dashed lines for types $3+4$. We only show the $3 \sigma$ error ellipse for the field data.

comparison, Fig. 6 shows type-dependent LFs for both field and cluster galaxies; the field LFs are normalized so that the overall field LF matches the overall cluster LF at $M_{b_{\mathrm{J}}}=-19$.

The $\chi^{2}$ tests show that the LFs for type 1 cluster and field galaxies are different over their entire range. This is due to the fact that the type 1 LF in clusters is both brighter and much steeper than in the field. The LFs of type 2 galaxies also differ, but Fig. 6 both shows that the difference is primarily due to the steeper faint-end slope; the $\chi^{2}$ fit shows that, to $M_{b_{\mathrm{J}}}<-18$, the two LFs match adequately. Finally, the LF of star-forming galaxies (types 3 and 4) are essentially identical in clusters and the field. These differences in the shapes of the type-specific LFs, and the different contributions from each type (as shown in Fig. 1), account for the differences between the overall cluster and field LFs. The steeper faint-end slopes for type 1 and type 2 galaxies in clusters may lead, at magnitudes fainter than those covered by our sample, to an upturn of the effective LF slope, such as has been claimed to exist in some clusters (De Propris \& Pritchet 1998).

One caveat concerns the assumption implicit in equation (9) that the galaxies without spectral types have the same type distribution as those for which types could be determined. In order to test the importance of this issue, we have computed LFs for each of the spectral types without any completeness correction. We find that $M^{*}$ is hardly changed whereas $\alpha$ is flattened by about 0.1 ; nevertheless, the LFs of type 1 and 2 galaxies remain steeper than in the field whereas types $3+4$ are consistent with the field LF. Incompleteness in spectral type comes from two sources: galaxies which were not observed and therefore have neither redshift nor spectral type and which are therefore an unbiased sample, and galaxies whose spectra have too low a signal-to-noise to yield a redshift or a spectral type. The untyped galaxies are roughly divided evenly between these two categories. It is only the latter category that may suffer from bias, in that galaxies of a specific type may be preferentially misidentified. We have therefore adopted a 'maximal correction' where all of these galaxies are assigned to each type in turn. This effect is small for types 1 and 2 but may be significant for types 3 and 4 . This is shown in Fig. 6. However, no matter what correction is applied, cluster galaxies of all types have LFs with faint-end slopes as steep or steeper than their counterparts in the field.

These differences between the type-dependent LFs in the cluster and field samples appear to be inconsistent with previous claims for a universal type-dependent LF (Binggeli, Sandage \& Tammann 1988; Jerjen \& Tammann 1997; Andreon 1998). However, it needs to be noted that these earlier studies are concerned with morphological types, which are only moderately well correlated with our spectral types (Fig. 1), so that a given spectral type may include a range of morphological types.

It is instructive at this stage to compare LF parameters for field and cluster galaxies, divided by spectral type. Fig. 7 shows that, progressing from late to early types, the cluster LFs have brighter $M^{*}$ and flatter $\alpha$, as observed in the field LFs (Madgwick et al. 2002). The trend in the value of $M^{*}$ with type is stronger in clusters than in the field, with a similar value of $M^{*}$ for type $3+4$ galaxies but a significantly brighter $M^{*}$ for type 1 galaxies. However, the trend of $\alpha$ with type is weaker for cluster galaxies than for field galaxies - at faint magnitudes the type $3+4$ LF is steeply rising $(\alpha=-1.3)$ in both clusters and the field, but the type 1 LF in the field is actually falling at faint magnitudes $(\alpha=-0.52)$ while in clusters it is merely flat $(\alpha=-1.05)$. This trend of $\alpha$ with environment for type 1 galaxies is apparent even within the cluster sample. The type 1 galaxies in richer clusters have a LF with a steeper faint end than those in poorer clusters, which in turn have a steeper LF than type 1 galaxies in the field (see Table 3 and Fig. 7), although this is only valid to $1.5 \sigma$. This is consistent with a tendency for the star formation rate to be broadly anticorrelated with the faint-end slope, which has been observed by Martinez et al. (2002) for galaxies in groups identified within the $2 \mathrm{dFGRS} 100 \mathrm{~K}$ release. Both Yagi et al. (2002) and Goto et al. (2002) claim similar variations in their samples.

\subsection{Cluster subsamples}

We have also compared subsamples of clusters chosen according to velocity dispersion, richness, B-M type and likelihood of containing substructure. Similarly, we also considered samples of galaxies within and outside two King core radii from the cluster centre. These results are summarized in Table 3 . We find that the values of $M^{*}$ and 


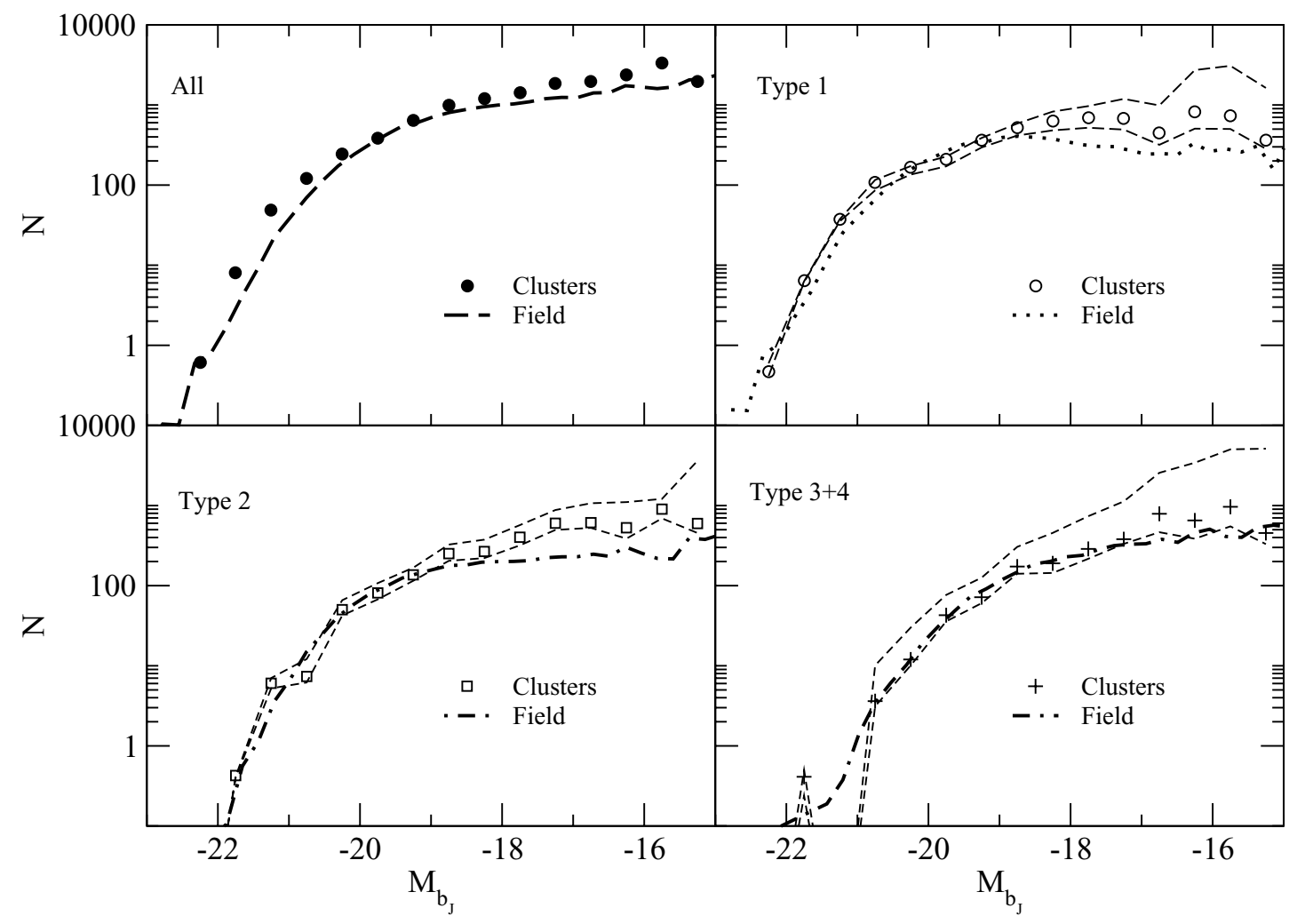

Figure 6. Comparison of cluster and field LFs. The open symbols are the type-completeness corrected cluster counts shown in Fig. 3. Thick lines represent the field LFs from Madgwick et al. (2002), normalized to agree with the cluster LF at $M_{b_{\mathrm{J}}}=-19$. The two thin lines show the luminosity distribution for no completeness correction (lower line) and for the maximal correction discussed in the text (upper line). Error bars are excluded for clarity (see Fig. 3 for error bars). The error ellipses are shown in Fig. 5 for both the field and cluster samples.

$\alpha$ for each pair considered (e.g. low and high velocity dispersion) do not differ at more than $1.5 \sigma$ in all cases. The only possible exception is for the case of clusters containing substructure, where $\alpha$ appears to be steeper. In all cases (again, excepting clusters with likely substructure) the derived LF parameters are consistent with those of the total LF within about $1.5 \sigma$. This is in contrast with Lumsden et al. (1997), who provided weak evidence for differences in the LFs of clusters with high and low $\sigma$, and with Valotto et al. (1997), who suggested differences between rich and poor clusters.

The difference in the LF of clusters with substructure is potentially interesting, as it is generally believed that substructure is an indicator of recent or ongoing cluster merging. However, an examination of the confidence contours for the fitted Schechter function parameters, shown in Fig. 4, shows that when the correlated nature of the parameters is considered, the fits to the LFs of the clusters with and without substructure are in fact consistent at better than $2 \sigma$.

The similarity of the LFs for the various subsamples is confirmed by two-sample $\chi^{2}$ tests, which indicate that the probabilities that the pairs of contrasted subsamples have consistent LFs are: 99.3 per cent for early and late B-M clusters, 35.6 per cent for high and low $\sigma$ clusters, 50.0 per cent for rich and poor clusters, 27.1 per cent for clusters with and without substructure, and 0.9 per cent for galaxies within and outside $300 \mathrm{kpc}$ from the cluster centre. Inspection of Fig. 4 shows that the LFs of galaxies in the inner $300 \mathrm{kpc}$ and the outer regions of clusters differ in detail; in particular, the inner region LF is a poor fit to the Schechter function, with a deficit of $L^{*}$ galaxies and an excess of brighter objects. This is reminiscent of the galactic cannibalism scenario of Ostriker \& Hausman (1978) and Malumuth
\& Richstone (1984), where $L^{*}$ galaxies are preferentially destroyed to fuel the growth of giant ellipticals, D and cD galaxies.

An alternative approach to quantifying the differences between LFs is to calculate the dwarf-to-giant $(\mathrm{D} / \mathrm{G})$ ratio. We define giants as galaxies with $-21.5<M_{b_{\mathrm{J}}}<-18$ and dwarfs as galaxies with $-18<M_{b_{\mathrm{J}}}<-16.0$, as in Driver et al. (1998a). These ratios are tabulated in Table 3 for all the LFs we consider. In general, our cluster samples have $\mathrm{D} / \mathrm{G}$ ratios comparable with those of moderately rich systems in Driver et al. (1998a). The D/G ratios for the contrasting pairs of subsamples only differ at the $\sim 1 \sigma$ level, except for clusters with and without substructure $(1.5 \sigma)$ and the inner and outer regions of clusters $(2.2 \sigma)$ - in no case is the difference in $\mathrm{D} / \mathrm{G}$ ratio highly significant.

The overall impression is therefore one of broad universality of the LF over a range of cluster properties. This is surprising if we consider the very considerable differences between the LFs of the various spectral types in the field (Madgwick et al. 2002), as different mixtures of types would lead to different LFs. For instance, the morphology-density relation might lead us to expect that rich and massive clusters would be more elliptical-rich than poor, low-mass clusters; likewise, early B-M clusters should be dominated by ellipticals, while late B-M type clusters should be spiral-rich. Mixing the field LFs of the different morphological mixes in these differing proportions would lead to clusters with significantly different LFs. The reason this does not appear to be the case is that the (spectral) type-specific LFs are more similar in clusters than they are in the field (see Fig. 5), so that changing the mixture of types within clusters has less effect than we might have expected. 

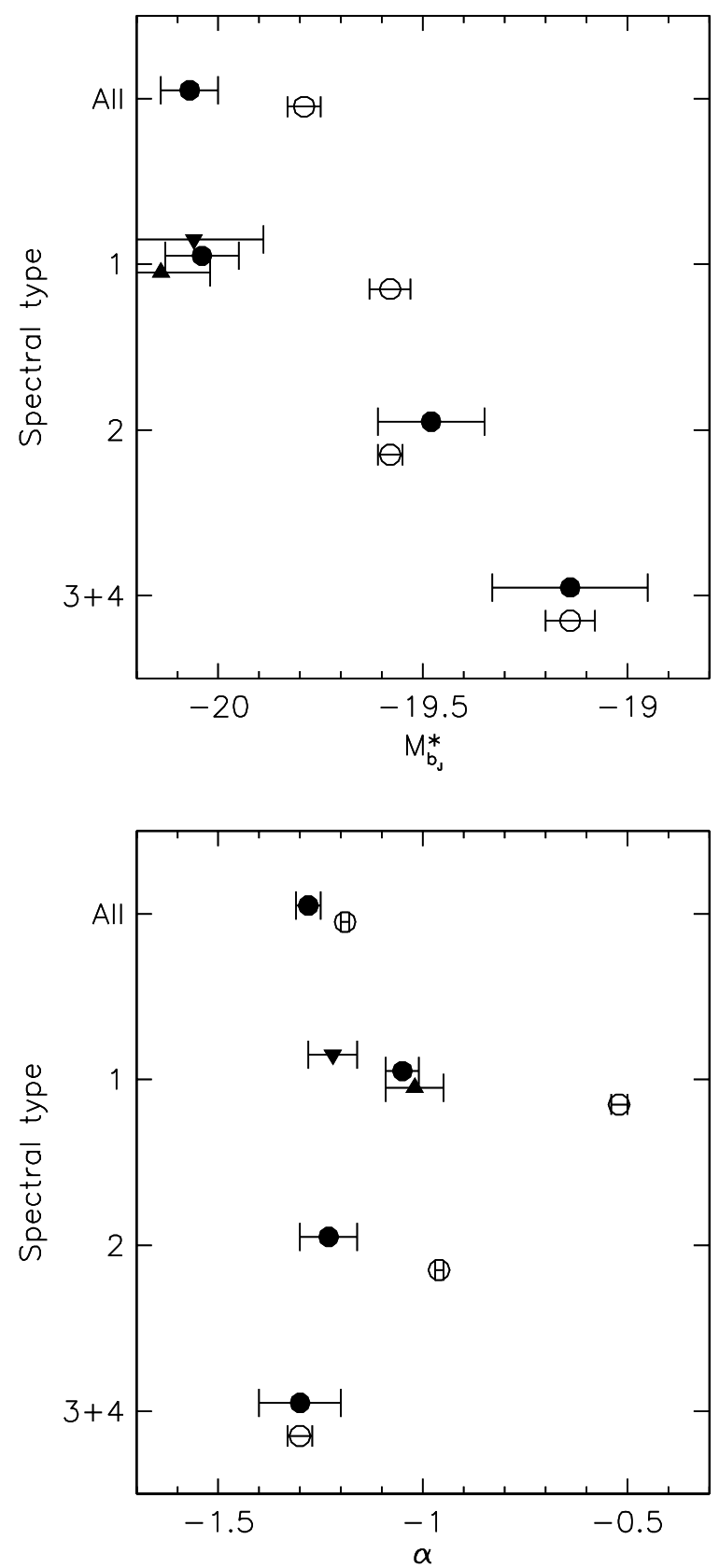

Figure 7. Comparison of cluster and field LF parameters. Open symbols are for field galaxies and filled symbols for cluster galaxies. Also shown are the LF parameters for type 1 galaxies in richer clusters (down-triangle) and poorer clusters (up-triangle).

\subsection{Implications for galaxy formation}

The main conclusions of our analysis are as follows:

(i) We have determined the composite LF of galaxies in clusters from the 2dFGRS. The LF is well fitted by a Schechter function with parameters $M_{b_{\mathrm{J}}}^{*}=-20.07 \pm 0.07$ and $\alpha=-1.28 \pm 0.03$. This is significantly different to the field LF of Madgwick et al. (2002), having a characteristic magnitude that is approximately 0.3 mag brighter and a faint-end power-law slope that is approximately 0.1 steeper.

(ii) There is no significant evidence for variations in the LF across a broad range of cluster properties; the LF appears similar for clus- ters with high and low velocity dispersions, for rich and poor clusters, for clusters with early and late B-M types, and for clusters with and without substructure. However, the core regions of clusters differ from the outer parts in having an excess of very bright galaxies.

(iii) Breaking down the LF by spectral type, the same trends are apparent in clusters as in the field: the LFs of earlier-type galaxies, with lower star formation rates, have brighter characteristic magnitudes and shallower faint-end slopes; the LFs of later-type galaxies, with higher star formation rates, have fainter characteristic magnitudes and steeper faint-end slopes. The trend in faint-end slope, which is the dominant difference between the LFs of the various spectral types in the field, is much less pronounced in clusters. The smaller differences between the LFs of different spectral types in clusters explain why variations in cluster properties giving rise to significant variations in the mixture of types do not lead to significant differences in the cluster LFs.

(iv) A comparison between the field and cluster LFs of each spectral type reveals that while the LF of late-type, star-forming galaxies is very similar in clusters and the field, the LF of earlytype galaxies with low star formation rates is both brighter and steeper in clusters than in the field; intermediate types in clusters have a LF with a similar bright end but a steeper faint end than their field counterparts. As early and intermediate spectral types are predominant in clusters, the overall cluster LF is also brighter and steeper than the overall field LF.

The above results may be compared with two recent redshiftbased studies of galaxy clusters. De Propris et al. (1998) derived the $K$-band LF of galaxies in the inner 25 arcmin of the Coma cluster from a 100 per cent complete sample of members, and found that the cluster LF is indistinguishable from the general field LF. Christlein $\&$ Zabludoff (2002) use a redshift survey of six clusters to derive $R$-band LFs for both the cluster members and the field galaxies in the foreground and background of the clusters. They also find that the $R$-band field and cluster LFs are very similar. These results are for samples selected in red and infrared passbands, and are therefore less sensitive to star formation, to which our $b_{\mathrm{J}}$-selected sample is more closely correlated. In contrast to these results, we find that there are small but statistically significant differences between field and cluster galaxies, with the blue-selected cluster LF being both brighter and steeper than that in the field. The similarity between the field and cluster LFs in the red and near-infrared passbands suggests that field and cluster galaxies with the same total stellar mass have similar integrated star-formation histories, while the difference between the field and cluster LFs in the blue simply means that the star formation rate is significantly affected by the cluster environment.

Trentham (1998) claimed that the LFs of nine clusters were very similar to each other and also to some field LFs. When this is compared with the field LF of Loveday et al. (1992) which is well fitted to the bright end, the cluster LF shows an excess at bright magnitudes. This is similar to our result, where most of the difference between field and cluster LFs comes from bright galaxies (the two LFs can be shown to be identical for $M<-20.5$ ).

Up until now it has been possible to claim that the observed differences between cluster and field LFs were due in large part simply to the different proportions in which supposedly universal type-specific LFs were mixed in the two environments. This explanation is now precluded by the finding that the type-specific LFs differ significantly with environment (in fact, as Fig. 5 shows, the difference between clusters and the field is less for the overall LF than for some of the type-specific LFs). 
Another way to understand the differences between the field and cluster LFs is to consider a simple 'closed-box' model. In this model we consider the type-specific field LFs to be the initial LFs within the volumes which today have collapsed to form the clusters. The relative normalizations of the LFs are based on the observed relative numbers of the different types in the field and in clusters (types 1:2:3+4 in the proportions 0.36:0.32:032 in the field and 0.54:0.24:0.22 in clusters; see Fig. 1) and the assumption that the total number of objects in the initial and final LFs is the same (i.e. neglecting mergers within the closed-box volume). Evolution from the initial (field) type-specific LFs to the final (cluster) type-specific LFs occurs almost entirely through processes which suppress star formation as the cluster collapses and becomes denser (Lewis et al. 2002), converting galaxies from later types to earlier types. In the simplest version of this model, this suppression of star formation does not affect the galaxies' luminosities. Fig. 8 shows the initial (field) and final (cluster) LFs of each spectral type in this naive model, and compares these LFs to the observed cluster LFs.

This model has some successes: it is consistent with the fact that the type $3+4$ cluster LF has the same shape but a lower normalization than the field LF, and it does cause the initial field LFs of the type 1 and 2 galaxies to evolve towards the forms they are observed to have in clusters. However, this passive steepening of the LFs, achieved by shifting galaxies from the steeper LFs of later types to the shallower LFs of earlier types, fails to steepen the LFs sufficiently to reproduce the observed clusters.

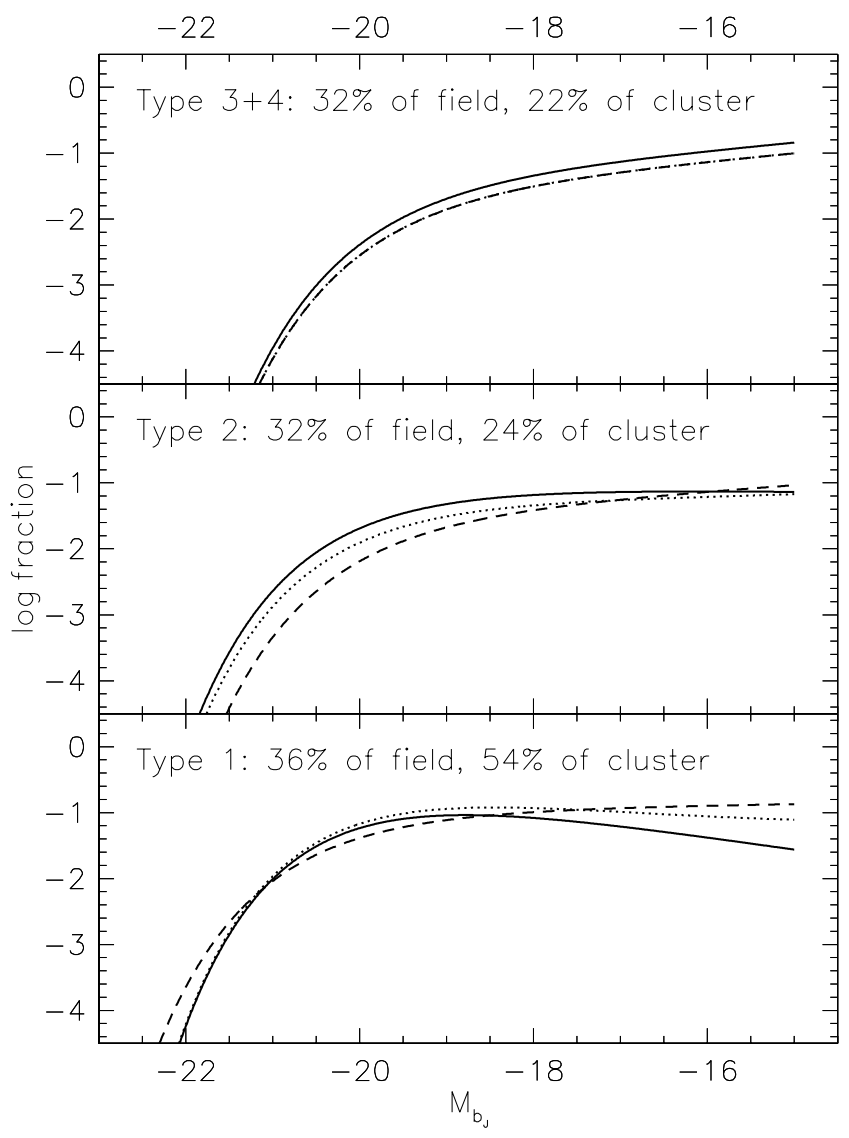

Figure 8. A comparison of the field LF (solid line) and closed-box model cluster LF (dotted line) to the observed cluster LF (dashed line) for each spectral type. Note that for type $3+4$ the dotted line is identical to the dashed line.
A more successful model requires both passive steepening as galaxies shift from later to earlier types and also active steepening due to luminosity-dependent fading. In such a model, the suppression of star formation affects about a third of type $3+4$ galaxies, independent of luminosity. The affected galaxies' instantaneous star formation rate decreases, lowering the strength of their $H \alpha$ emission so that they become type 2 galaxies. This leaves the shape of the type $3+4$ LF the same, but reduces the numbers of these strongly star-forming galaxies. When type 2 galaxies undergo further suppression of their star formation rate, this has the effect of decreasing their $b_{\mathrm{J}}$ luminosities and eventually converting them to type 1 , where further fading may occur. This fading must be greater for brighter galaxies in order to actively steepen the LF slopes and reproduce the observed cluster LFs.

In addition, some merging (or cannibalism) is required to explain the small number of very bright type 1 galaxies in clusters which are not present in the field LFs, and may also help explain the deficit of bright type 2 and $L^{*}$ type 1 cluster galaxies. This is consistent with the conclusions of Christlein \& Zabludoff (2002), who find that the only difference between the $R$-band LFs of cluster and field galaxies is an excess of bright non-star-forming galaxies. However, the higher D/G ratio in the LFs of earlier-type galaxies in clusters indicates that suppression of star formation is more important than mergers in shaping the cluster LF.

It should be noted that the field LF is in fact the mean LF of the entire galaxy population, and is therefore dominated by galaxies belonging to groups. Thus, our results therefore imply that suppression of star formation is the dominant effect in evolving from the typical group environment to the rich cluster environment. Merger effects are expected to be more important in groups, however, and this will be investigated in a future paper based on the group catalogue derived directly from the $2 \mathrm{dFGRS}$.

The closed-box model is oversimplified in a variety of ways, and can only serve as a qualitative guide to understanding the processes shaping the LF of cluster galaxies. Unfortunately, a quantitative interpretation of our results is hampered by the fact that few theoretical studies have considered the evolution of the LF and its dependence on environment, and most have been limited to the brighter cluster members (e.g. Malumuth \& Richstone 1984). Detailed semianalytic models are clearly required to explore the relative importance of the various mechanisms that may be driving the evolution of galaxies in clusters. These models will need to be complemented by more stringent observational constraints to distinguish the effects of the different processes involved. It will be particularly important to obtain the near-ultraviolet and near-infrared LFs for large spectroscopic samples of cluster galaxies. These will yield both the instantaneous star formation rate and the total stellar mass of the galaxies, and reveal where galaxies are evolving due to mergers and where they are undergoing changes in their star formation rate.

\section{ACKNOWLEDGMENTS}

We would like to thank the referee, Stefano Andreon, for a number of suggestions which have improved the content of this paper. We also thank Daniel Christlein and Ann Zabludoff for having shown us their results in advance of submission. RDP and WJC acknowledge funding from the Australian Research Council for this work. We are indebted to the staff of the Anglo-Australian Observatory for their tireless efforts and assistance in supporting $2 \mathrm{dF}$ throughout the course of the survey. We are also grateful to the Australian and UK time assignment committees for their continued support for this project. 


\section{REFERENCES}

Abell G. O., 1958, ApJS, 3, 211

Abell G. O., Corwin H. C., Olowin R., 1989, ApJS, 70, 1

Adami C., Mazure A., Katgert P., Biviano A., 1998, A\&A, 336, 63

Adami C., Ulmer M. P., Durret F., Nichol R. C., Mazure A., Holden B. P., Romer A. K., Savine C., 2000, A\&A, 353, 930

Andreon S., 1998, A\&A, 336, 98

Binggeli B., Sandage A., Tammann G. A., 1988, ARA\&A, 26, 509

Blanton M. R. et al., 2001, AJ, 121, 2358

Christlein D., Zabludoff A. I., 2002, ApJ, submitted

Colless M. M., 1989, MNRAS, 237, 799

Colless M. M. et al., 2001, MNRAS, 328, 1039

Cross N. et al., 2001, MNRAS, 324, 825

Dalton G. B., Maddox S. J., Sutherland W. J., Efstathiou G., 1997, MNRAS, 289, 263

De Propris R., Pritchet C. J., 1998, AJ, 116, 1118

De Propris R., Eisenhardt P. R., Stanford S. A., Dickinson M., 1998, ApJ, 503, L45

De Propris R. et al., 2002, MNRAS, 329, 87

Dressler A., 1978, ApJ, 223, 765

Driver S. P., Couch W. J., Phillipps S., 1998a, MNRAS, 301, 369

Driver S. P., Couch W. J., Phillipps S., Smith R., 1998b, MNRAS, 301, 357

Fitchett M., 1988, MNRAS, 230, 161

Folkes S. et al., 1999, MNRAS, 308, 459

Gaidos E. J., 1997, AJ, 113, 117

Garilli B. M., Maccagni D., Andreon S., 1999, A\&A, 342, 408

Goto T. et al., 2002, PASJ, 54, 515

Jerjen H., Tammann G. A., 1997, A\&A, 321, 713
Kennicutt R. C., 1992, ApJS, 79, 255

Lewis I. J. et al., 2002, MNRAS, 334, 673

Lopez-Cruz O., Yee H. K. C., Brown J. P., Jones C., Forman W., 1997, ApJ, 475, L97

Loveday J., Peterson B. A., Maddox S., Efstathiou G., 1992, ApJ, 390, 338

Lugger P. M., 1986, ApJ, 303, 535

Lugger P. M., 1989, ApJ, 343, 572

Lumsden S. L., Nichol R. C., Collins C. A., Guzzo L., 1992, MNRAS, 258, 1

Lumsden S. L., Collins C. A., Nichol R. C., Eke V. R., Guzzo L., 1997, MNRAS, 290, 119

Madgwick D. S. et al., 2002, MNRAS, 333, 133

Malumuth E. M., Richstone D. O., 1984, ApJ, 276, 413

Martinez H. J., Zandivarez A., Merchán M. E., Dominguez M. J. L., 2002, MNRAS, 337, 1441

Norberg P. et al., 2002, MNRAS, 337, 1068

Ostriker J. P., Hausman M. A., 1978, ApJ, 224, 320

Paolillo M., Andreon S., Longo G., Puddu E., Gal R. R., Scaramella R., Djorgovski G., de Carvalho R., 2001, A\&A, 367, 59

Rauzy S., Adami C., Mazure A., 1998, A\&A, 337, 31

Schechter P., 1976, ApJ, 203, 279

Small T. A., Sargent W. L. W., Hamilton D., 1997, ApJ, 487, 512

Solanes J. M., Salvador-Solé E., González-Casado G., 1999, A\&A, 343, 733

Trentham N., 1998, MNRAS, 294, 193

Valotto C. A., Nicotra M. A., Muriel H., Lambas D. G., 1997, ApJ, 479, 90

Yagi M., Kashikawa N., Sekiguchi M., Doi M., Yasuda N., Shimasaku K., Okamura S., 2002, AJ, 123, 87

This paper has been typeset from a $\mathrm{T}_{\mathrm{E}} \mathrm{X} / \mathrm{LT} \mathrm{E} \mathrm{X}$ file prepared by the author. 